\title{
Enhancing In Vivo Exposure in the Treatment of Panic Disorder and Agoraphobia Using Location-Based Technologies: A Case Study
}

\author{
Ignacio Miralles ${ }^{*}(\mathbb{D}$, Carlos Granell I*, \\ Azucena García-Palacios ${ }^{1 *}$, Diana Castilla ${ }^{2,3 *}$, \\ Alberto González-Pérez 1*, Sven Casteleyn 1*, \\ and Juana Bretón-López ${ }^{\text {** }}$
}

\begin{abstract}
Panic disorder (PD) is quite prevalent and often appears along with agoraphobia (PD/A). The treatment of choice is cognitive behavioral therapy (CBT). Transdiagnostic intervention, an emotion-focused, cognitive behavioral intervention that has led to the Unified Protocol (UP), emphasizes the common underlying mechanisms that contribute to the development and maintenance of emotional disorders such as PD/A. A core feature of this treatment approach is in vivo exposure (IVE) to feared situations, which aims to prevent avoidance behaviors and encourages the patient to confront feared situations gradually. It is a difficult component for patients, especially when implementing the exposure on their own. Different feedback formats can be used to increase adequate IVE and reduce overt or subtle avoidance. The use of smartphones is a very useful option to initiate and sustain exposure behavior. The purpose of this study is to describe the use of location-based technologies (LBTs) during the IVE component of the UP treatment of a 47-year-old patient with PD/A. The acceptability and usability of the system were assessed. The Symptoms platform was employed during the exposure module, using LBT with a smartphone app. The patient reported positive expectations, high satisfaction scores, and an overall satisfactory experience. Enhancing key therapeutic components during treatment through the development of media-based tools is a very promising future research aim, and the possibility of using advanced smartphone features should be explored.
\end{abstract}

\section{Keywords}

panic disorder, agoraphobia, UP, location-based technologies, exposure

\footnotetext{
'Universitat Jaume I, Castellón de la Plana, Spain

¿Universitat de València, Spain

IInstituto Salud Carlos III, Santiago de Compostela, Spain

*All authors contributed equally to this work.
}

\section{Corresponding Author:}

Ignacio Miralles, Department of Computer Languages and Systems, Universitat Jaume I, Avenida de Vicent Sos Baynat, s/n, I207I Castellón de la Plana, Spain.

Email: mirallei@uji.es 


\section{Theoretical and Research Basis for Treatment}

\section{Panic Disorder and Agoraphobia}

The Diagnostic and Statistical Manual of Mental Disorders (5th ed.; DSM-5; American Psychiatric Association [APA], 2013) defines panic disorder (PD) as recurrent and unexpected panic attacks followed by concerns about their consequences for physical or social aspects. The estimated 12-month prevalence of PD in adults and adolescents across the United States and several European countries is around 2\% to 3\% (Bélanger et al., 2017). PD has a high comorbidity with agoraphobia disorder, with agoraphobic symptoms estimated to affect between $33 \%$ and $50 \%$ of patients with PD. Agoraphobia disorder is described as experiencing a marked fear or anxiety triggered by real or anticipated exposure to a wide range of situations (APA, 2013).

Such disorders are frequently associated with a high social impact (avoidance of relevant personal events), occupational impairment (absence from work or school), and physical disability (comorbidity with numerous general medical conditions). In addition, PD entails considerable socioeconomic costs and the highest number of medical visits of all the anxiety disorders, with the strongest effects in the presence of agoraphobia. The aforementioned symptoms, which are linked to the diagnosis, are clearly associated with poorer quality of life (APA, 2013).

\section{Cognitive Behavioral Therapy and the Unified Protocol}

The treatment of choice for $\mathrm{PD}$ and agoraphobia $(\mathrm{PD} / \mathrm{A})$ is cognitive behavioral therapy (CBT), which is considered a first-line treatment (McHugh et al., 2009). The efficacy of this treatment is supported by The Task Force Report on Empirically Supported Treatments (ESTs; Chambless \& Hollon, 1998) and the 12th Division of the APA. It has been further reinforced by meta-analysis efficacy studies that report important and long-lasting improvements (Hofmann et al., 2012). The core feature of CBT is in vivo exposure (IVE) to feared situations, which aims to prevent avoidance behaviors and make the patient confront the feared situations gradually with the goal of habituation. This component is well established, and it is used to disconfirm misappraisals and eliminate conditioned emotional responses to external conditions in agoraphobic situations linked to the disorder. Despite the efficacy of CBT and IVE, it is a difficult component for patients, especially when implementing the exposure on their own, which reveals the inherent difficulties of the exposure component for people suffering from PD/A (Olatunji et al., 2009). Therefore, it becomes important to enhance IVE and its motivational features with some kind of therapeutic support.

Traditionally, guidelines for IVE sessions have included the support of a co-therapist during the exposure, or even the presence of the therapist outside of the therapy office (Gloster et al., 2011). In addition, different feedback formats have also been used to increase adequate IVE and reduce overt or subtle avoidance, which helps the patient (Powers et al., 2004). However, some of these measures can have potential costs in terms of time and interference, whereby patients turn the measures into safety signals (Gloster et al., 2011). Furthermore, new developments in psychopathology have increased the emphasis on the common underlying mechanisms that contribute to the development and maintenance of multiple disorders, especially emotional disorders (as in the case of PD/A), and novel strategies are being designed to target these disorders (Mansell $\&$ McEvoy, 2017). This is the case of the transdiagnostic intervention, an emotion-focused, cognitive behavioral intervention that has led to the Unified Protocol (UP) as a form of CBT to help patients learn new ways to respond to their emotions (Barlow et al., 2010). A number of studies (Dear et al., 2016; Farchione et al., 2012), including important randomized controlled trial (RCT) studies, have shown that the transdiagnostic approach and the UP can be as effective as the traditional CBT diagnostic approach (Barlow et al., 2010). In addition, reviews and meta-analysis have reported promising results (Newby et al., 2015; Pearl \& Norton, 2017). 


\section{Technology-Associated Psychological Interventions}

In recent years, there has been an increased interest in using technology-supported psychological interventions as a form of treatment for emotional disorders, including PD/A. Several studies have been conducted to improve the effectiveness or clinical utility of psychological treatments. The first promising technology was virtual reality (VR), which emerged as an effective tool for applying IVE, reporting efficacy in the treatment of anxiety disorders (Opriş et al., 2012), and more specifically, PD/A (Botella et al., 2007; Choi et al., 2005; Pérez-Ara et al., 2010). These pioneering studies included the CBT approach. Furthermore, media development has led to the use of the internet to deliver CBT, obtaining very adequate results in systematic reviews, and meta-analysis studies confirming the efficacy of these computerized programs (Newby et al., 2016). In addition, new approaches, such as the previously mentioned transdiagnostic intervention, have been tested in RCT studies as effective treatment options in a web-based format for affective and mood disorders (Newby et al., 2016; Tulbure et al., 2018).

The implementation of Information and Communication Technologies (ICTs) for psychological intervention has been empirically tested. Along with the technology itself and the clinical effectiveness, the issue of acceptability of the technology-guided treatment is important when considering whether or not the use of ICT will affect therapeutic adherence and outcomes (Santana \& Fontenelle, 2011). Acceptability refers to the degree to which users are satisfied or at ease with the service and willing to use it (Rush \& Scott, 2004), and it influences whether a treatment is perceived as fair and reasonable, appropriate, and nonintrusive in addressing a problem (Campos et al., 2018; Wallin et al., 2016). In the Technology Appraisal Guidance from the United Kingdom, it is considered a priority to properly assess intervention acceptability in treatments and their related variables; expectations, satisfaction, and usability. Thus, these variables become crucial features in psychotherapy results (Greenberg et al., 2006). Several studies have focused on expectations and satisfaction using different ICT tools, such as VR or internet-based treatment to deliver psychological treatments, and specifically IVE, in mental disorders and PD/A (Botella et al., 2007; Quero et al., 2014), reporting high satisfaction with the exposure component when mediated by technologies. Studying the acceptability and usability of an ICT requires taking into account a very specific conceptualization of the user experience. The aim is to enhance the ICT's development by having a specific user explore the product's ease of use within a clearly defined context and with clearly marked goals. Even though few studies have addressed this topic (Anderson et al., 2007; Botella et al., 2016; Campos et al., 2018), the aforementioned ICTs have been shown to be well supported in terms of acceptability issues.

\section{Location-Based Technologies Through the Use of a Smartphone App}

In mental health, additional advantages can be obtained through the use of ICT, such as mobile phones and smartphones. Smartphone use has experienced a huge increase in recent years (Taylor $\&$ Silver, 2019). Consequently, researchers have started focusing on the impact of these devices when used as tools to implement psychological interventions and IVE components (Rizvi et al., 2011). Smartphone portability could be very useful in a variety of feared situations in which $\mathrm{PD} / \mathrm{A}$ symptoms arise. Additional smartphone features such as location-based technologies (LBTs) capable of tracking the patient's position could be a starting point to initiate and sustain the exposure behavior. As a result, they can lead to an improvement in IVE by increasing patients' motivation and commitment. Although LBT could become a tool when prescribing IVE, the patient would still have to carry out the exposure on his or her own. This study identified a set of advantages of using smartphones: (a) the therapist can ensure that the patient is actually in the 
exposure situation; (b) the therapist can ensure that the patient stays in the exposure situation for the necessary time to fulfill the goals; and (c) the patient's motivation and commitment can be enhanced in the real exposure environment. In previous studies, when carrying out the treatment, the context has been considered and treated as a variable. In the study by Addepally and Purkayastha (2017), the authors looked at using a mobile application (app) that would monitor the location of depressed people. The app detected whether depressed patients were in uncrowded areas (a frequent trait in depressed individuals according to the authors) and, if so, the patient would receive therapeutic strategies and self-help assessments through notifications. In a case report study (Olbrich et al., 2016), a patient suffering from obsessive-compulsive disorder was allowed to configure alarms in case he or she was in the same place for a long time, using an app that tracked his or her position. However, to the best of our knowledge, there are no reports on LBT in a smartphone application for the treatment of PD/A disorder during IVE. With all this in mind, the purpose of the current case study is to describe the use of LBT in an IVE component during the treatment of a patient diagnosed with severe $\mathrm{PD} / \mathrm{A}$ and have the patient assess the acceptability and usability of the system.

\section{Case Introduction}

The patient was a 47-year-old married male who worked as a sports trainer. He met the Diagnostic and Statistical Manual of Mental Disorders, fifth edition (DSM-5) criteria for panic and agoraphobia disorder. When the patient arrived at our clinic, he had significant agoraphobic avoidance, even though he did not experience unexpected full panic attacks because he avoided every feared situation. The patient presented comorbidity with acrophobia.

\section{Presenting Complaints}

The patient rated both his problem's severity and its interference as an 8 (on a scale ranging from 0 to 10). The main physical sensations experienced by the patient included hand sweating and dizziness. The patient stated that he was extremely concerned about the possibility of suffering panic attacks in a feared situation, which could produce the onset of physical symptoms that could result in loss of control. This problem created additional issues in his professional life because he felt he could not move far away from his home town.

\section{History}

The patient had previously received treatment for this problem, but discontinued it because he did not perceive any improvement. Although he does not remember exactly when his problem began, he reported having the problem since he was a child, probably starting with a fear of heights that evolved to other situations. The problem became progressively worse, to the point of severely interfering in his life, until he sought help in our clinic. Due to his problem, several of his job tasks had been impaired, resulting in the loss of main work activities. In terms of medication, he had been taking $1 \mathrm{mg}$ of alprazolam daily for years, and he reported that it helped him sleep. The patient reported no history of substance abuse or misuse.

\section{Assessment}

Several common psychiatric testing measures were used to establish the diagnosis and evaluate the effects of the intervention. However, given that the component under study was IVE with LBTs (IVE+LBT), only the measures related to this component will be presented in this article (with the exception of the diagnostic measures). 


\section{Measures for the Diagnosis and Target Behaviors}

- Anxiety Disorders Interview Schedule-IV (ADIS-IV; Di Nardo et al., 1993). The ADIS-IV is a semi-structured interview that assesses anxiety disorders included in the DSM. The tool has demonstrated interrater reliability ranging from satisfactory to excellent when used by expert clinicians who are familiar with the DSM diagnostic criteria. In this study, the PD and agoraphobia and specific phobia sections were used.

- Fear and avoidance scales (adapted from Marks \& Mathews, 1979). The patient and the therapist established situations that the patient avoided because of his PD/A and that created considerable impairment in the patient's daily life. He rated the level of avoidance on a scale from 0 to $10(0=$ I never avoid it; $10=$ I always avoid it $)$ and the level of fear on a scale from 0 to $10(0=$ no fear; $10=$ extreme fear $)$ for each target situation.

\section{Measures for Expectations and Satisfaction With the ICT System}

- Expectation and satisfaction scale for the IVE+LBT component used previously in our research group in other ICT contexts (Botella et al., 2009; Quero et al., 2014). This questionnaire was used to measure the patient's expectations before the IVE+LBT component and satisfaction after it. The questionnaire included six items: how logical the IVE+LBT component seemed, to what extent it could satisfy the patient, whether the patient would recommend this component treatment to others, whether it would be useful in treating other problems, the component's usefulness for the patient's problem, and to what extent it could be invasive. This last item was considered a key factor in assessing the ICT system during IVE. Due to the main characteristics of the LBT, it was important to assess any disruption, annoyance, or intrusion in the patient's privacy caused by the ICT. Both parts (expectations and satisfaction) ranged from 0 to $10(0=$ not at all; $10=$ very much $)$.

\section{Measures for Acceptability and Usability of the ICT System}

- System Usability Scale (Brooke, 1996) is one of the most widely used tools for assessing perceived usability (Albert \& Tullis, 2013). It consists of 10 items, half written in a direct style and the other half in an inverse style. A 5-point scale is used to rate the level of agreement, from 1 (strongly disagree) to 5 (strongly agree). This scale has a score contribution of the scale position minus 1 for Items 1, 3, 5, 7 and 9, and 5 minus the scale position for Items $2,4,6,8$ and 10. A formula is used to calculate the score as a percentage from 0 to 100 .

\section{Case Conceptualization}

The patient received the UP for Transdiagnostic Treatment of Emotional Disorders delivered by the UP Institute (Center for Anxiety and Related Disorders [CARD], Boston University). The treatment program was structured in eight modules. The sessions included in each module lasted around $1 \mathrm{hr}$ each and were delivered weekly. The IVE+LBT component was carried out during the interoceptive and situational exposure module, in line with the structure of the UP program (Barlow et al., 2019). The patient was gradually exposed to his main target behaviors based on the exposure hierarchy he created with the therapist, progressing from the easiest to the most challenging situations. LBT was used during the IVE.

\section{System Description}

The application used with the case study was developed in 2018 as part of an ongoing platform called Symptoms. The goal of the Symptoms platform is to create personalized, location-based 


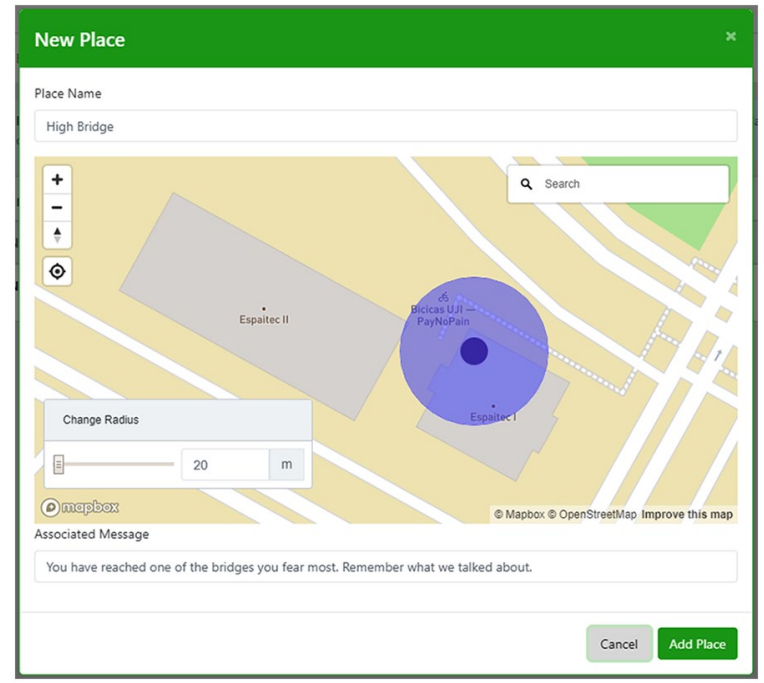

Figure I. Screenshot of the new place form.

mobile applications for mental health treatments. The platform presents three different tools: a web-based Builder, the generated mobile app, and a web-based Viewer.

Builder. The therapist accesses the web-based Builder through an URL, using widely supported web browsers. After being registered and identified, the therapist can create patient profiles and applications. To create a patient profile, the therapist introduces some basic descriptive data. To create an application for the patient, the therapist must enter some basic data and then select the relevant places for the patient on a map. For each of these places, she or he has to enter a personalized message (Figure 1). Once the app has been created, the patient receives an Application Key (AppKey) that is used to activate the mobile application. This application can be updated by the therapist using the Builder, without any further action of the patient but to open the application.

App. The outcome of the Builder tool is an Android-based mobile application. The patient downloads it on her or his mobile phone from the Google Play Store and installs it. To launch it the first time, an AppKey, which is provided by the therapist, is requested. Once it is entered, the app is unlocked and runs in the background, without interfering with the patient's regular use of the smartphone. Once the app is running, the user's location is continuously captured and every time the patient arrives at any of the configured places, she or he will receive a notification with the personalized message.

Viewer. The therapist can access the web-based Viewer through the same URL as the Builder. Once authenticated, a therapist can browse and analyze her or his patient's gathered data. She or he can also inspect the gathered data on the spatial and temporal dimensions by observing the places visited by the patient over time (Figure 2).

The whole platform is developed by integrating a combination of technologies. Figure 3 depicts the architecture of the platform. The Builder and the Viewer are part of the same web application, developed with the JavaScript Angular framework version 6. They are connected to a Google Firebase Firestore database for data persistence, and both of them utilize the Firebase's authentication system. The mobile application is developed in Java using the Android Studio 


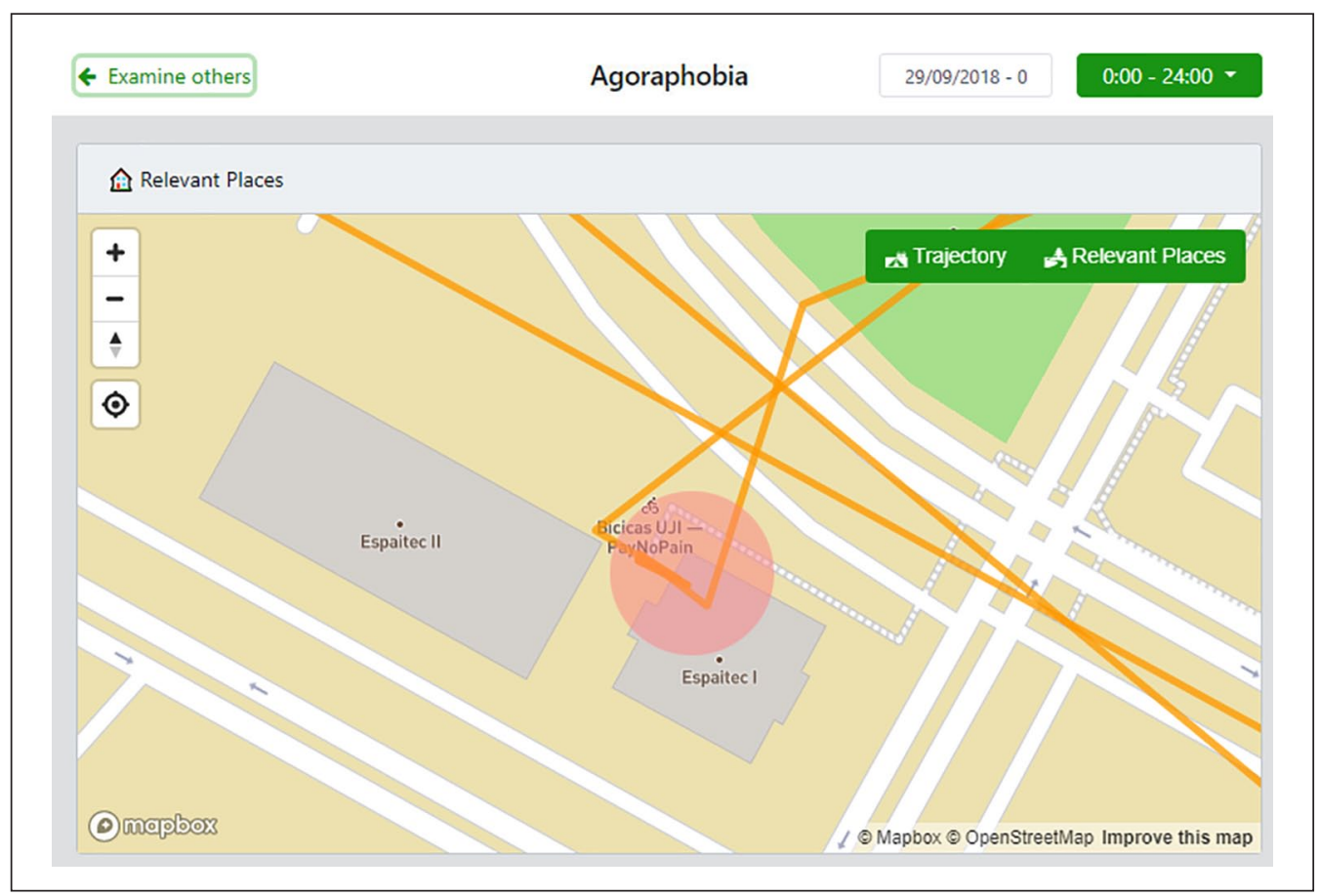

Figure 2. Screenshot of the Viewer.

development environment, and it connects to the same Firestore database as the web-based tools. Google's location-based services are used to gain access and communicate with different embedded sensors, such as accelerometer, GPS, or Wi-Fi. In this way, the current location of the patient is established.

\section{Course of Treatment and Assessment of Progress}

\section{Therapist}

The therapist was a member of our research team with more than 10 years of experience in the therapeutic field, including both technology-mediated and psychological interventions.

\section{Course of Treatment}

The patient asked for help at the Jaume I University Anxiety Disorders Clinic, Spain. He underwent a screening assessment and, having met the inclusion criteria, signed a consent form to participate in the study. Inclusion criteria included the following: (a) meeting the panic and agoraphobia diagnostic criteria and (b) providing written, informed consent. Exclusion criteria included the following: (a) suffering from a severe mental disorder (schizophrenia, bipolar disorder, and alcohol and/or substance dependence disorder); (b) having a medical disease/condition that keeps her or him from carrying out the psychological treatment; and/or (c) receiving another psychological treatment during the study. The assessment consisted of two 60-min sessions to establish the diagnosis and the target behaviors. The ADIS-IV interview was carried out in the first session, and the second session was used to conduct other self-report measures and establish the patient's target behaviors. The rest of the measures linked to the UP treatment were carried 


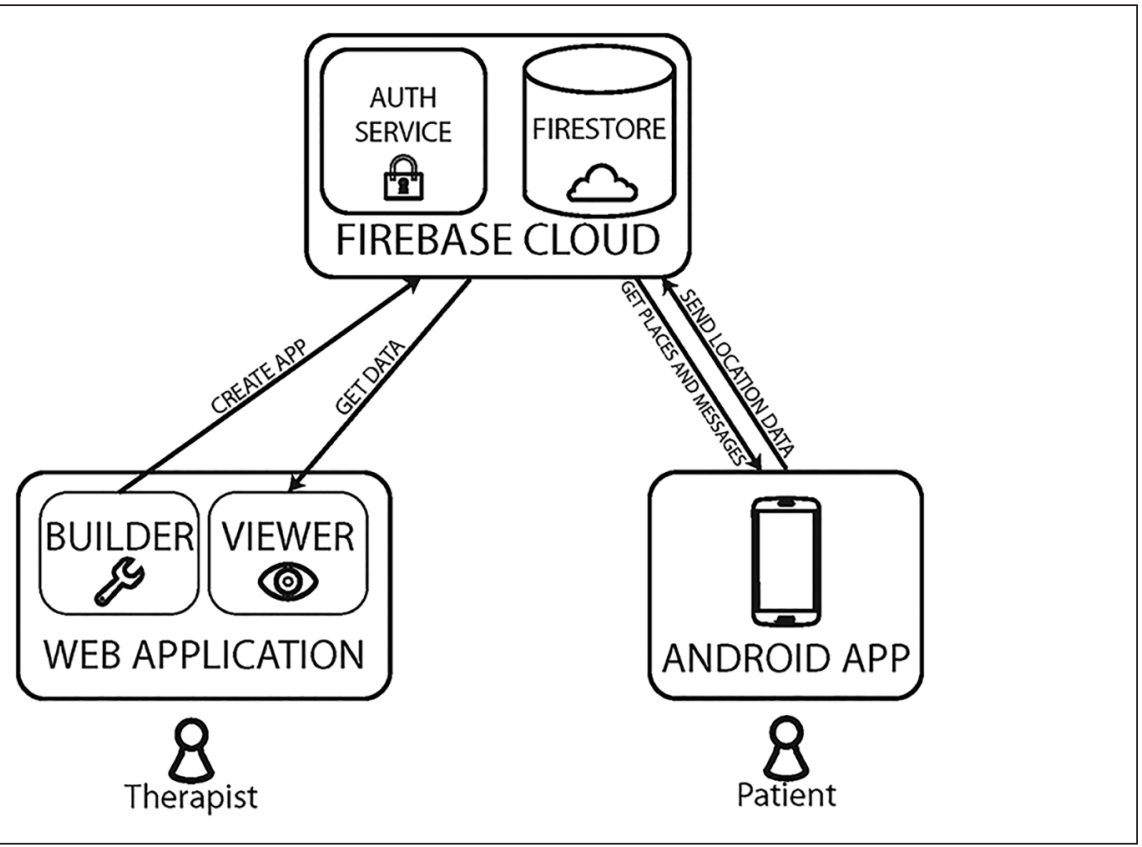

Figure 3. Technological architecture of the Symptoms platform.

out in the context of the full therapy. Given that the component under study was the IVE+LBT, only the measures related to this component will be presented in this article and emphasized in this procedure section. Following the assessment and before starting the treatment, the therapist explained the basis of the treatment and the use of LBT to support the IVE during the exposure module. The patient agreed to take part in the research.

Once the consent form for participating in the research and the consent form to use the LBT had been filled out properly, the treatment started. The patient received the UP for Transdiagnostic Treatment of Emotional Disorders (UP Institute, CARD, Boston University), with a total of eight modules. The sessions included in each module lasted about $1 \mathrm{hr}$. The patient carried out weekly IVEs for a period of 4 months. The exposure sessions were conducted in correspondence with the UP modules. Before starting the exposure module, therapist and patient created a gradual exposure hierarchy based on the core situations feared by the patient. Then, the expectations about the IVE+LBT component were evaluated by the patient before the first exposure task was conducted. The locations assessed by the fear and avoidance scales were configured, their positions were set by the therapist using the Symptoms Builder, and the app was installed on the patient's smartphone. Every time the patient arrived at one of the core places during the IVE tasks, he received a notification with the particular message written by the therapist "you have just arrived at an important place. Remember to work with all the strategies you know." The LBT was used during the patient's IVE tasks, in which he had to confront the different scenarios related to his fear and his main target behaviors in a gradual exposure hierarchy, progressing from the easiest to the most challenging situations. Following all the exposure tasks, the satisfaction, acceptability, and usability of the IVE+LBT were assessed by the patient on the self-report measures.

\section{Results}

Figure 4 displays the main target behaviors selected for the exposure tasks, as well as the fear and avoidance ratings from the patient. As the figure shows, the target behaviors were bridge, 


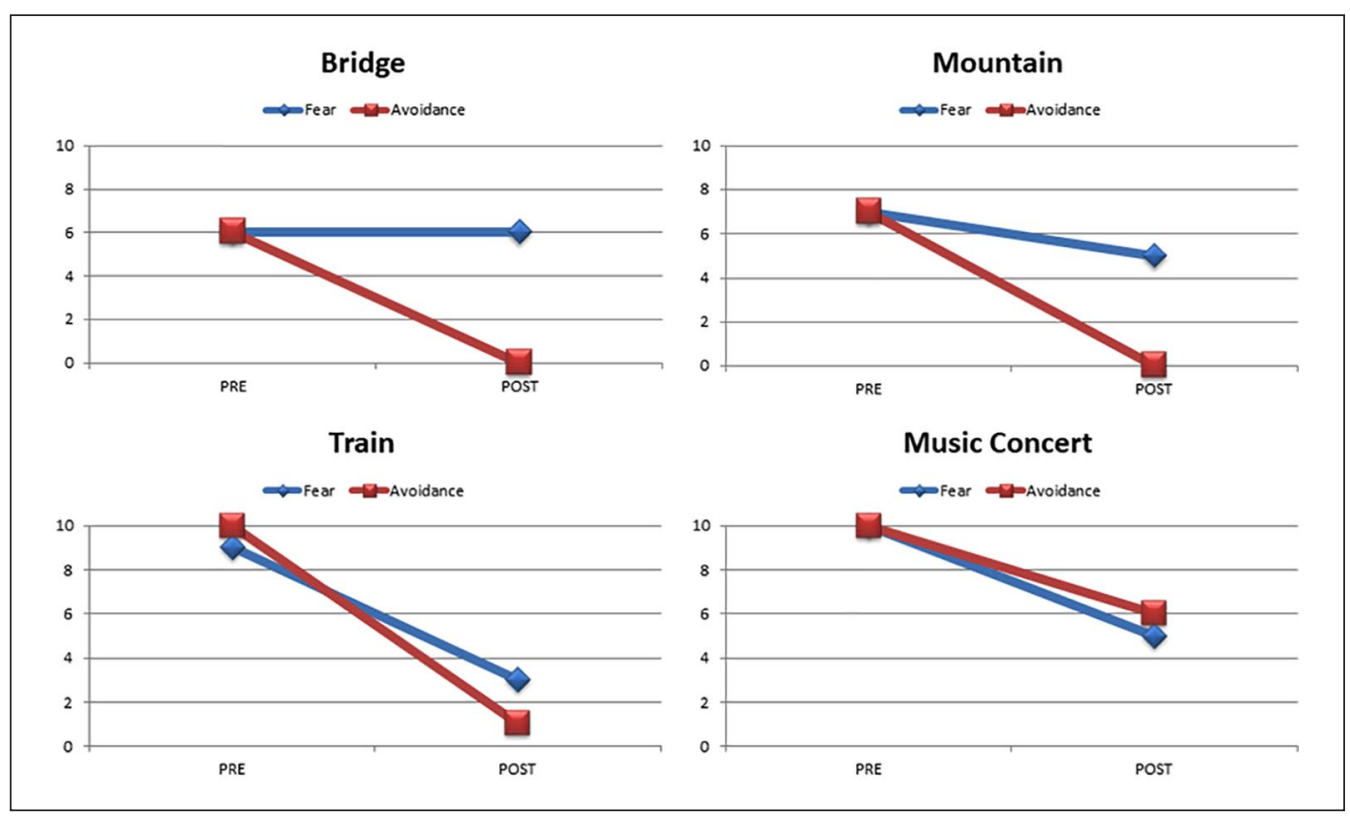

Figure 4. Located target behaviors with fear and avoidance rating.

mountain, train, and music concert. All the selected target behaviors caused a certain level of impairment in different areas of the patient's life.

The locations were retrieved by the LBT and the app was configured to receive the notification when the patient arrived at these core places during exposure tasks. Once the exposure session had started, the patient achieved $100 \%$ of the goals established with regard to the exposure tasks, that is, there was no avoidance in any case at the end of the exposure session. Even though it was not a goal of this study, preliminary results for the fear and avoidance situations show an important reduction in the score on the scheduled target behaviors rated by the patient and positioned by the IVE+LBT.

\section{Expectations and Satisfaction With the IVE + LBT}

Regarding the patient's acceptance of the treatment, he reported high expectations before starting the IVE+LBT component. The scores on Items 1 to 5 (logic, satisfaction, recommendation, usefulness for others, and usefulness for this problem) were 10, whereas Item 6 (invasive) scored 0. Data show that the patient's acceptance of the treatment before starting it was good. In addition, he indicated a highly positive evaluation afterward, giving Items 1 to 5 a score of 10 and Item 6 a score of 0 . The question of how invasive the technology could be was especially important for this research. The patient rated this question as "not at all" before and after using the system. The loss of privacy due to sending detailed information about the system use to the therapist could have created interference with the opinion about the system. However, this was not the case in this study.

\section{Satisfaction With and Usability of the IVE + LBT}

The perceived satisfaction and usability for the user was very high on all the items, except Item 4 (see Table 1). This item refers to the following statement "I think I would need the support of a technical person to be able to use this system." An interview with the user revealed that this score 
Table I. IVE+LBT Usability Test.

Item I: I think I would like to use this system frequently 5

Item 2: I found the system unnecessarily complex

Item 3: I thought the system was easy to use

Item 4: I think that I would need the support of a technical person to be able to use this system

Item 5: I found that the various functions in this system were well integrated

Item 6: I thought there was too much inconsistency in this system

Item 7: I would imagine that most people would learn to use this system very quickly 5

Item 8: I found the system very cumbersome to use

Item 9: I felt very confident using the system

1

Item 10: I needed to learn a lot of things before I could get going with this system

was due to technical problems experienced one day during exposure tasks. This problem was related to the frequency with which the mobile phone made the GPS request. The Android operating system's energy management component applies particular policies about the use of background services. By default, the system slows down processes with high energy usage to reduce battery consumption, and in some cases, this can delay the app's geolocation service, causing a delay in message delivery. Once the problem was detected, the background service was partially modified to improve its execution precision (making the application request energy management permission at the beginning of its use). With these changes, the messages worked properly again.

The overall score for satisfaction and usability was 95 points, which, according to the qualitative scale developed by Bangor et al. (2008), means that the system is within an acceptable range, with adjectives ranging between "excellent" and "best imaginable."

Finally, some qualitative information was collected from the patient's experience. The following are some of the opinions expressed by the user after the IVE+LBT component:

General opinion: "The use of the system was very interesting for me, especially in the motivational aspect. It was extremely important for me to have a record of the completed exposures." Specific experiences: "There was a day when I forgot the smartphone with the app, and I went back in order to carry out the exposure task. The most important aspect about the system, one I really appreciated, was the role it took on as a personal trainer for each exposure task."

Regarding the notification: "Ok, the notification could be interesting, but it was not the most important thing for me. Recording my activity and recording the work well done, that was what made me even more committed throughout the treatment."

\section{Complicating Factors}

The patient began treatment with complicated and severe PD and agoraphobia. Although he made significant improvements in several core situations in his daily functioning, some factors complicated the treatment process. His busy daily work routine proved to be an important difficulty for him, affecting his attendance at some therapy sessions and his ability to complete homework assignments. Therapist and patient adapted the schedule to complete the weekly sessions as much as possible. Despite these complicating factors, the patient was dedicated to the treatment and willing to engage in the different therapeutic components. With regard to the technical side of the ICT used in the study, some problems with the LBT system were experienced one day during the exposure tasks. This issue has been discussed in the "Course of Treatment and Assessment of Progress" section of the results. The technical problem was detected, improvements were implemented, and after these changes, the messages worked properly again. 


\section{Access and Barriers to Care}

There were no significant concerns about access or barriers to care. Regarding the costs, therapy was conducted at a University Anxiety Disorders Clinic, in the context of a research project where access to psychological care is free. Fortunately, the patient was able to perform multiple IVEs, for example, taking the train, and assume the associated cost, for example, the price of the train tickets. In relation to the attitudes about mental health services, the patient showed distrust because he had previously received treatment for this problem but had not perceived any improvement. However, ICT inclusion in the psychological treatment and the use of LBT to support the IVE caught his attention. Consequently, the patient agreed to take part in the research.

\section{Follow-Up}

Brief appointments took place after the end of the IVE sessions, and some emails and phone calls were made to contact him and obtain more information about his psychological status. His daily routine has not allowed the patient to maintain frequent and systematic exposure to the feared situations. However, both face-to-face appointments and email or phone communication showed the patient's positive progress. The patient even planned a train trip by himself during the summer period. He could not remember having had the desire to travel (by train) in more than 20 years. Follow-ups after the summer period have been agreed on.

\section{I Treatment Implications of the Case}

The results obtained in this case study show that the use of LBT has relevant implications for the treatment of $\mathrm{PD} / \mathrm{A}$. There seem to be several positive consequences of monitoring the IVE sessions in real time during the patient's treatment: (a) the certainty that the patient's efforts are being recorded and visualized by the therapist; (b) the fact that the technology is well accepted by the patient; and (c) the possibility of increasing adherence and commitment to the scheduled IVE tasks. The patient reported positive expectations and high satisfaction scores, coinciding with previous studies showing that ICT treatments are well accepted by different population (Gómez et al., 2018; Potter et al., 2015; Quero et al., 2014). These results using more advanced technology are quite important, given that a positive relationship between expectations and satisfaction with ICT treatments and intervention efficacy was found in Mira et al. (2019). In addition, the data on intrusiveness are especially relevant. As pointed out earlier, the patient reported that the IVE+LBT was not invasive before or after the treatment, and that it did not interfere with the fulfillment of the exposure tasks. Finally, usability results reported the patient's satisfactory experience with the system. Based on the technology acceptance model, the authors suggested that one of the factors that can be related to the intention to use a product in the future is ease of use (Carvalho et al., 2012; Huang \& Liao, 2015). Therefore, usability, which is already an important attribute in the use of any technology, becomes a prerequisite for the use of technology for mental health. Technology must be completely transparent from the beginning; otherwise, a slow learning curve or high frustration during use might interfere with the therapy and negatively influence the outcome of the treatment. Therefore, efforts to research and ensure the usability of new ICT approaches are crucial.

Previous research has focused on the ethical considerations of using exposure therapy (Olatunji et al., 2009) and the fact that it may become problematic in different stages of the therapy outside the safe context with the therapist or co-therapist. The development of specific ICT strategies as additional tools to guide the IVE can be useful in reducing avoidance, increasing overall functioning, and enhancing the patient's motivation and commitment to critical components of the therapy and, in this case, feared situations. In sum, some of these tool-guided exposure elements could significantly improve adherence to treatment and reduce dropout rates (a major issue 
encountered in research studies), particularly in PD/A, which is highly characterized by avoidance behaviors, with attrition rates from 10\% to 55\% (Santana \& Fontenelle, 2011; White et al., 2010) and disruptive variables of the disorder that can affect the treatment's discontinuation.

From a technical point of view, and given the simplicity of the application's creation using the Symptoms Builder, the adaptability of this tool is also presented as a positive feature. Changing the indicated places or the information delivered can be done quickly and without specific technical knowledge. This makes it easy to adapt the application to the evolution of the patient or for use with others, bypassing the technological barrier involved in customizing applications for different patients, and even for therapists. Generating this type of in situ intervention has always been somewhat complex, requiring time-consuming traditional methods or trusting the patient to carry out the indicated tasks. Thanks to this tool, this process has been streamlined, allowing the therapists to focus her or his attention on the therapeutic content and delegating the monitoring and delivery of the materials to the mobile device.

With regard to the application itself, at the moment, it only allows a basic configuration: places and information for the associated notifications in text format. However, it would have been interesting to be able to include other variables, for example, different messages arriving depending on the time (a message at the beginning of the exposure, a message in the middle, and one at the end) or more complex content such as multimedia. Currently, only text information is allowed, but moving forward, it would be interesting to include images or videos to improve the quality of the intervention. Although the application is focused on intervention, it would also have been positive to use real-time feedback in the form of questionnaires that are able to identify, for example, the patient's anxiety level. In general, there is a series of functions that are not very complex from a technological point of view, but would make it possible to considerably improve the options when creating the application.

In terms of the study's shortcomings, the main one is that it is a case study. To increase the confidence in the described IVE+LBT system and maximize the new exposure format for PD/A and other mental disorders, it would be necessary to apply this technology to larger samples and robust studies with an experimental design that includes a control group. Despite these limitations, we believe that this work contributes to the improvement of IVE for PD/A through innovative ICT tools such as LBT.

\section{2 Recommendations to Clinicians and Students}

The IVE+LBT system described in the present research could represent a useful starting point and prove essential in extending its use to several additional disorders, especially those related to core locations involved in the course of the disorder and the treatment. Examples of disorders that could benefit from this system are situational phobias, where the system can play a similar role in enhancing the IVE tasks, and gambling disorders, where LBTs could be used to enhance stimulus control during the initial treatment of this nonsubstance-related disorder, thus reducing abstinence violations. At the present time, our team is already working on the first pilot study using this technology for this latter type of population (Diaz et al., 2019). We consider this line of research to be particularly relevant for future studies that want to evaluate the role of these devices in treatment motivation, patient commitment during exposure tasks, and therapeutic adherence. Future studies could also address the inclusion of assessment tools in the system. That is, to advance in the Ecological Momentary Assessment (EMA), the inclusion of the anxiety and avoidance level, and even ratings of beliefs in catastrophic thoughts, could be carried out through the same platform, creating an electronic record that could offer a full picture of the IVE task performed by the patient. The results of this study suggest that using the application to continue to add intervention components during IVE exposure tasks, following scheduled ways of administering psychological support, as in previous studies (Oromendia et al., 2016), can be very beneficial for the patient, helping to reduce dropout rates and increase treatment adherence. 
The design of these novel strategies to target psychological disorders and enhance key therapeutic components could benefit from mobile and LBTs and constitute very promising future research aims. Smartphone devices also offer innovative advantages and so it is imperative to continue to explore new possibilities.

\section{Declaration of Conflicting Interests}

The author(s) declared no potential conflicts of interest with respect to the research, authorship, and/or publication of this article.

\section{Funding}

The author(s) disclosed receipt of the following financial support for the research, authorship, and/or publication of this article: I.M. was funded by the Spanish Ministry of Education, Culture, and Sports (grant reference FPU14/00020). A.G.-P. was funded by the Spanish Ministry of Education, Culture, and Sports (grant reference FPU17/03832). C.G. and S.C. were funded by the Ramon y Cajal Programme of the Spanish government (grant references RYC-2014-16913 and RYC-2014-16606, respectively). This study was supported by funding from 16I336.01/1; programa UJI-A2016-14, Jaume I University and by the SyMptOMS project, funded under the "Programa Estatal de I+D +i Orientada a los Retos de la Sociedad" of the Spanish government (reference RTI2018-099939-BI-00).

\section{ORCID iD}

Ignacio Miralles (iD) https://orcid.org/0000-0002-1205-5434

\section{References}

Addepally, S. A., \& Purkayastha, S. (2017). Mobile-application based cognitive behavior therapy (CBT) for identifying and managing depression and anxiety. In Digital human modeling. Applications in health, safety, ergonomics, and risk management: Health and safety (pp. 3-12). Springer, Cham.

Albert, W., \& Tullis, T. (2013). Measuring the user experience: Collecting, analyzing, and presenting usability metrics. Elsevier.

American Psychiatric Association. (2013). Diagnostic and statistical manual of mental disorders (5th ed.). American Psychiatric Publishing.

Anderson, P., Zimand, E., Schmertz, S. K., \& Ferrer, M. (2007). Usability and utility of a computerized cognitive-behavioral self-help program for public speaking anxiety. Cognitive and Behavioral Practice, 14(2), 198-207.

Bangor, A., Kortum, P. T., \& Miller, J. T. (2008). An empirical evaluation of the system usability scale. International Journal of Human-Computer Interaction, 24(6), 574-594.

Barlow, D., Farchione, T., Fairholme, C., Ellard, K., Boisseau, C., Allen, L., \& Ehrenreich-May, J. (2010). Unified protocol for transdiagnostic treatment of emotional disorders: Therapist guide. Oxford University Press.

Barlow, D., Farchione, T., Fairholme, C., Ellard, K., Boisseau, C., Allen, L., \& Ehrenreich-May, J. (2019). Protocolo unificado para el tratamiento transdiagnóstico de los trastornos [Unified protocol for transdiagnostic treatment of emotional disorders] emocionales (J. Osma and E. Crespo, Trans.). Alianza Editorial.

Bélanger, C., Courchesne, C., Leduc, A. G., Dugal, C., El-Baalbaki, G., Marchand, A., \& Perreault, M. (2017). Predictors of dropout from cognitive-behavioral group treatment for panic disorder with agoraphobia: An exploratory study. Behavior Modification, 41(1), 113-140.

Botella, C., Gallego, M. J., Garcia-Palacios, A., Baños, R. M., Quero, S., \& Alcañiz, M. (2009). The acceptability of an internet-based self-help treatment for fear of public speaking. British Journal of Guidance \& Counselling, 37(3), 297-311.

Botella, C., García-Palacios, A., Villa, H., Baños, R. M., Quero, S., Alcañiz, M., \& Riva, G. (2007). Virtual reality exposure in the treatment of panic disorder and agoraphobia: A controlled study. Clinical Psychology \& Psychotherapy, 14(3), 164-175.

Botella, C., Mira, A., Moragrega, I., García-Palacios, A., Bretón-López, J., Castilla, D., . . Baños, R. M. (2016). An internet-based program for depression using activity and physiological sensors: Efficacy, expectations, satisfaction, and ease of use. Neuropsychiatric Disease and Treatment, 12, 393-406. 
Brooke, J. (1996). SUS: A “quick and dirty” usability scale. In P. W. Jordan, B. Thomas, B. A. Weerdmeester, \& A. L. McClelland (Eds.), Usability evaluation in industry (pp. 189-194). Taylor \& Francis.

Campos, D., Mira, A., Bretón-López, J., Castilla, D., Botella, C., Baños, R. M., \& Quero, S. (2018). The acceptability of an internet-based exposure treatment for flying phobia with and without therapist guidance: Patients' expectations, satisfaction, treatment preferences, and usability. Neuropsychiatric Disease and Treatment, 14, 879-892.

Carvalho, M. L. D., Guimarães, H., Ferreira, J. B., \& Freitas, A. (2012). Intention to use M-learning: An extension of the technology acceptance model. In XXXVI Encontro da ANPAD.

Chambless, D. L., \& Hollon, S. D. (1998). Defining empirically supported therapies. Journal of Consulting and Clinical Psychology, 66(1), 7-18.

Choi, Y. H., Vincelli, F., Riva, G., Wiederhold, B. K., Lee, J. H., \& Park, K. H. (2005). Effects of group experiential cognitive therapy for the treatment of panic disorder with agoraphobia. Cyberpsychology, Behavior, and Social Networking, 8(4), 387-393.

Dear, B. F., Staples, L. G., Terides, M. D., Fogliati, V. J., Sheehan, J., Johnston, L., \& Titov, N. (2016). Transdiagnostic versus disorder-specific and clinician-guided versus self-guided internet-delivered treatment for social anxiety disorder and comorbid disorders: A randomized controlled trial. Journal of Anxiety Disorders, 42, 30-44.

Diaz, L., Miralles, I., Granell, C., Bretón-López, J., González-Pérez, A., Casteleyn, S., \& Gacía-Palacios, A. (2019). Enhancing stimulus control in the treatment of gambling disorder using location-based technologies. In The 6th ESRII conference. Copenhagen.

Di Nardo, P. A., Moras, K., Barlow, D. H., Rapee, R. M., \& Brown, T. A. (1993). Reliability of DSMIII-R anxiety disorder categories: Using the Anxiety Disorders Interview Schedule-Revised (ADIS-R). Archives of General Psychiatry, 50(4), 251-256.

Farchione, T. J., Fairholme, C. P., Ellard, K. K., Boisseau, C. L., Thompson-Hollands, J., Carl, J. R., \& Barlow, D. H. (2012). Unified protocol for transdiagnostic treatment of emotional disorders: A randomized controlled trial. Behavior Therapy, 43(3), 666-678.

Gloster, A. T., Wittchen, H. U., Einsle, F., Lang, T., Helbig-Lang, S., Fydrich, T., \& Arolt, V. (2011). Psychological treatment for panic disorder with agoraphobia: A randomized controlled trial to examine the role of therapist-guided exposure in-situ in CBT. Journal of Consulting and Clinical Psychology, 79(3), 406-420.

Gómez, I., Flujas-Contreras, J. M., Ruiz-Castañeda, D., \& Castilla, D. (2018). A virtual reality-based psychological treatment in long-term hospitalization: A case study. Clinical Case Studies, 18, 3-17.

Greenberg, R. P., Constantino, M. J., \& Bruce, N. (2006). Are patient expectations still relevant for psychotherapy process and outcome. Clinical Psychology Review, 26(6), 657-678.

Hofmann, S. G., Asnaani, A., Vonk, I. J. J., Sawyer, A. T., \& Fang, A. (2012). The efficacy of cognitive behavioral therapy: A review of meta-analyses. Cognitive Therapy and Research, 36(5), 427-440.

Huang, T. L., \& Liao, S. (2015). A model of acceptance of augmented-reality interactive technology: The moderating role of cognitive innovativeness. Electronic Commerce Research, 15(2), 269-295.

Mansell, W., \& McEvoy, P. M. (2017). A test of the core process account of psychopathology in a heterogenous clinical sample of anxiety and depression: A case of the blind men and the elephant? Journal of Anxiety Disorders, 46, 4-10.

Marks, I. M., \& Mathews, A. M. (1979). Brief standard self-rating for phobic patients. Behaviour Research and Therapy, 17(3), 263-267.

McHugh, R. K., Smits, J. A. J., \& Otto, M. W. (2009). Empirically supported treatments for panic disorder. Psychiatric Clinics of North America, 32(3), 593-610.

Mira, A., Soler, C., Alda, M., Baños, R., Castilla, D., Castro, A., . . Botella, C. (2019). Exploring the relationship between the acceptability of an internet-based intervention for depression in primary care and clinical outcomes: Secondary analysis of a randomized controlled trial. Frontiers in Psychiatry, 10, Article 325.

Newby, J. M., McKinnon, A., Kuyken, W., Gilbody, S., \& Dalgleish, T. (2015). Systematic review and meta-analysis of transdiagnostic psychological treatments for anxiety and depressive disorders in adulthood. Clinical Psychology Review, 40, 91-110.

Newby, J. M., Twomey, C., Li, S. S. Y., \& Andrews, G. (2016). Transdiagnostic computerised cognitive behavioural therapy for depression and anxiety: A systematic review and meta-analysis. Journal of Affective Disorders, 199, 30-41. 
Olatunji, B. O., Deacon, B. J., \& Abramowitz, J. S. (2009). The cruelest cure? Ethical issues in the implementation of exposure-based treatments. Cognitive and Behavioral Practice, 16(2), 172-180.

Olbrich, H., Stengler, K., \& Olbrich, S. (2016). Smartphone based geo-feedback in obsessive compulsive disorder as facilitatory intervention: A case report. Journal of Obsessive-Compulsive and Related Disorders, 8, 75-78.

Opriş, D., Pintea, S., García-Palacios, A., Botella, C., Szamosközi, Ş., \& David, D. (2012). Virtual reality exposure therapy in anxiety disorders: A quantitative meta-analysis. Depression and Anxiety, 29(2), 85-93.

Oromendia, P., Orrego, J., Bonillo, A., \& Molinuevo, B. (2016). Internet-based self-help treatment for panic disorder: A randomized controlled trial comparing mandatory versus optional complementary psychological support. Cognitive Behaviour Therapy, 45(4), 270-286.

Pearl, S. B., \& Norton, P. J. (2017). Transdiagnostic versus diagnosis specific cognitive behavioural therapies for anxiety: A meta-analysis. Journal of Anxiety Disorders, 46, 11-24.

Pérez-Ara, M., Quero, S., Botella, C., Baños, R., Andreu-Mateu, S., García-Palacios, A., \& Bretón-López, J. (2010). Virtual reality interoceptive exposure for the treatment of panic disorder and agoraphobia. Studies in Health Technology and Informatics, 154, 77-81.

Potter, C. M., Jensen, D., Kinner, D. G., Tellez, M., Ismail, A., \& Heimberg, R. G. (2015). Single-session computerized cognitive behavioral therapy for dental anxiety: A case series. Clinical Case Studies, 15, 3-17.

Powers, M. B., Smits, J. A. J., \& Telch, M. J. (2004). Disentangling the effects of safety-behavior utilization and safety-behavior availability during exposure-based treatment: A placebo-controlled trial. Journal of Consulting and Clinical Psychology, 72(3), 448-454.

Quero, S., Pérez-Ara, M. Á., Bretón-López, J., García-Palacios, A., Baños, R. M., \& Botella, C. (2014). Acceptability of virtual reality interoceptive exposure for the treatment of panic disorder with agoraphobia. British Journal of Guidance \& Counselling, 42(2), 123-137.

Rizvi, S. L., Dimeff, L. A., Skutch, J., Carroll, D., \& Linehan, M. M. (2011). A pilot study of the DBT coach: An interactive mobile phone application for individuals with borderline personality disorder and substance use disorder. Behavior Therapy, 42(4), 589-600.

Rush, B., \& Scott, R. E. (2004). Approved telehealth outcome indicator guidelines: Quality, access, acceptability and cost. Health Telematics Unit, University of Calgary.

Santana, L., \& Fontenelle, L. F. (2011). A review of studies concerning treatment adherence of patients with anxiety disorders. Patient Preference and Adherence, 5, 427-439.

Taylor, K., \& Silver, L. (2019). Smartphone ownership is growing rapidly around the world, but not always equally. https://www.pewresearch.org/global/2019/02/05/smartphone-ownership-is-growing-rapidlyaround-the-world-but-not-always-equally/

Tulbure, B. T., Rusu, A., Sava, F. A., Sălăgean, N., \& Farchione, T. J. (2018). A web-based transdiagnostic intervention for affective and mood disorders: Randomized controlled trial. JMIR Mental Health, $5(2)$, e 36.

Wallin, E. E. K., Mattsson, S., \& Olsson, E. M. G. (2016). The preference for internet-based psychological interventions by individuals without past or current use of mental health treatment delivered online: A survey study with mixed-methods analysis. JMIR Mental Health, 3(2), e25.

White, K. S., Allen, L. B., Barlow, D. H., Gorman, J. M., Shear, M. K., \& Woods, S. W. (2010). Attrition in a multicenter clinical trial for panic disorder. Journal of Nervous and Mental Disease, 198(9), 665-671.

\section{Author Biographies}

Ignacio Miralles is a PhD Student at the Geospatial Technologies Research Group at UJI, Spain. His research work focuses on developing tools to improve mental health.

Carlos Granell is a PostDoctoral Researcher at the Geospatial Technologies Research Group at UJI, Spain. His work focuses on spatial analysis, urban computing and interdisciplinary GIS.

Azucena García-Palacios is Full professor and the director of the Labpsitec group at UJI, Spain. His research work focuses on clinical psychology.

Diana Castilla is professor and researcher of the Labpsitec group at UV, Spain. His research work focuses on clinical psychology. 
Alberto González-Pérez is currently working toward the Ph.D. degree at the Geospatial Technologies Research Group at UJI, Spain. His research work focuses on mobile computing, cloud computing and IoT.

Sven Casteleyn is a PostDoctoral Researcher at the Geospatial Technologies Research Group at UJI, Spain. His work focuses on developing tools for improve mental health.

Juana Bretón-López is an associate professor and researcher of the Labpsitec group at UJI, Spain. His research work focuses on clinical psychology. 